\title{
Os dermique de tortue terrestre en Afrique du nord
}

\section{Colette Roubet}

\section{OpenEdition}

Journals

Édition électronique

URL : https://journals.openedition.org/encyclopedieberbere/2839

DOI : 10.4000/encyclopedieberbere.2839

ISSN : 2262-7197

\section{Éditeur}

Peeters Publishers

\section{Édition imprimée}

Date de publication : 2 juin 2013

Pagination : 5873-5881

ISBN : 978-2-7584-0194-0

ISSN : 1015-7344

\section{Référence électronique}

Colette Roubet, "Os dermique de tortue terrestre en Afrique du nord », Encyclopédie berbère [En ligne], 36 | 2013, document 032, mis en ligne le 12 mars 2021, consulté le 17 février 2022. URL : http:// journals.openedition.org/encyclopedieberbere/2839; DOI : https://doi.org/10.4000/ encyclopedieberbere.2839

Ce document a été généré automatiquement le 17 février 2022.

(c) Tous droits réservés 


\title{
Os dermique de tortue terrestre en Afrique du nord
}

\author{
Colette Roubet
}

1 On traitera ici de l'os dermique après une présentation détaillée du rôle de l'os* dans la composition des outillages préhistoriques. Des études focalisées sur l'animal, son éthologie et sa valeur d'exemple montrent comment s'est développé et répandu durant l'Holocène l'intérêt des populations pastorales pour cette créature modeste, dissimulée, non agressive, vivant en colonies dans des biotopes appropriés. On doit à la fréquentation de ces biotopes par des pasteurs leur découverte, leur collecte et surtout des créations inattendues, en relation peut-être avec des pratiques ou des cérémonies que suggère l'ocre* présent.

2 L'enveloppe dermique formant la carapace protectrice de la tortue terrestre est assez peu connue et aurait pu rester inexploitée. Les préhistoriens ont signalé la présence de ce reptile parmi les documents fauniques de l'Holocène en Afrique du Nord, sans s'attarder à son rôle secondaire dans la subsistance, mais en se penchant plutôt sur de petits éléments perforés, vestiges de pendeloques, présents ici comme en Europe, à la même époque. Ces curieux objets servirent de parure. Peu fréquents, associés à des contextes néolithiques, ils apparaissent dans des sites atlasiques et telliens. Leur présence a été soulignée par H. Camps-Fabrer en 1960 dans son ouvrage sur les Parures des temps préhistoriques en Afrique du Nord, C. Roubet (1966) leur a consacré une étude générale recensant 36 sites. Ces ornements parmi tant d'autres, prestigieux, auraient pu rester au catalogue des bribes anecdotiques d'une documentation curieuse. N'étaient les découvertes majeures faites au Damous* el-Ahmar dans les Némencha* et au Khanguet* Si Mohamed Tahar, dans la grotte Capéletti de l'Aurès* ouvrant de nouvelles perspectives (Roubet 1968, 1979).

3 Le pouvoir bénéfique et symbolique de ces ornements n'avait pourtant pas manqué d'être souligné, sans toutefois reconnaître à l'animal vivant un statut de même nature. Celui-ci est aujourd'hui retenu parce qu'il aurait pu avoir réservé à l'animal une capture particulière, ni ordinaire, ni au hasard, mais fondée sur l'acquisition et la transmission de ce pouvoir, en fonction du choix de la taille et de l'état de l'individu 
capturé, et dont l'expert en os dermique pourrait ensuite avoir bénéficié en exploitant ce matériau, puis ceux plus tard qui détiendraient les objets façonnés. Alors que cette chaîne de bienfaits transmis s'esquissait, surgit une démarche nouvelle.

4 La démarche globale et progressive adoptée part du biotope naturel pour s'approcher au plus près de l'animal vivant, de l'artisan préhistorique, des ses créations, dont une au moins deviendra pendeloque. Elle vivifie, comme nous le verrons plus loin, notre compréhension de la sphère symbolique, suggère le transfert de pouvoirs bénéfiques par le truchement d'actes et de gestes spéciaux, confortant ainsi l'hypothèse d'un rituel sous-jacent, passé inaperçu, qui serait resté insaisissable sans les réponses aux questionnements essentiels sur les motivations initiales: où, quand, qui, comment, pourquoi?

5 Connues aujourd'hui ces réponses sont étroitement liées au comportement pastoral des communautés du Néolithique* atlasique, préagricole. En effet, durant l'Holocène ancien-moyen, lors des transhumances hivernales, on sait que des communautés atlasiques de bergers quittèrent avec leurs troupeaux leurs abris enneigés d'altitude, pour séjourner jusqu'au printemps sur les piémonts et au bord des lagunes ; là, près des bassins fermés de la Merdja, au nord des Némencha (pour les pasteurs du Damous elAhmar), et non loin des lagunes d'eau douce du Tarf, au nord de l'Aurès (pour les pasteurs des grottes Capéletti, Bou Zabaouine, du Dj. Marshel, etc.), sur ces territoires attractifs, propices aux collectes diverses (oeufs d'autruche notamment), des générations de pasteurs capturèrent des tortues terrestres. Ces biotopes naturels conservaient encore au $\mathrm{XX}^{\mathrm{e}}$ siècle de petites colonies de tortues terrestres appréciées des populations locales, non loin d'Aïn Fakroun [= «Source de la tortue »]!

Comprendre comment passer de la capture de l'animal à la pendeloque a exigé des connaissances anatomiques de référence (documents fournis en 1965 par la Faculté des Sciences de l'Université d'Alger) pour pouvoir déterminer le matériel préhistorique ostéologique. L'intérêt de cette matière spéciale, pour les hommes préhistoriques, serait resté énigmatique sans la découverte au Damous el-Ahmar d'abord de plus de 300 pièces (Roubet 1966), et dans la grotte Capeletti de l'Aurès, de près de 600 pièces in situ et en stratigraphie, correspondant à tous les éléments constitutifs d'une carapace. Conservés dans de bonnes conditions, ces documents permettent de réunir toutes les parties anatomiques aboutissant à la reconnaissance d'une exploitation totale, et sur place, en montagne, (loin de biotopes naturels), de plusieurs dizaines d'individus (fig. 1 à 4).

7 L'anatomie nous servit donc de guide comme à l'artisan qui forma le projet d'exploiter une carapace vidée. Ce n'est qu'après cuisson dans l'eau bouillante et retrait des parties molles consommables (rituellement peut-être) que l'artisan disposa d'un ensemble osseux à exploiter. L'objectif d'une reconstitution des phases techniques permit de découvrir comment l'artisan obtint une pendeloque ; cette première enquête aboutit à la mise en place d'une chaîne opératoire complète. Restaient incomprises et hors du schéma d'une pendeloque quantité d'autres lamelles osseuses dessoudées, dont la face interne se trouvait zébrée d'incisions transversales (fig. 3). Cette seconde enquête aboutit à reconstituer une seconde chaîne opératoire pour obtenir la création d'une coupe ou d'une coupelle.

8 Retrouver les étapes d'un savoir faire spécial créant une pendeloque à partir du plastron ventral et une coupe à partir du bouclier dorsal, permit d'approcher de très près cette anatomie originale composée de plaques articulées, matière première 
présentant de nombreuses contraintes. On en tira la conclusion qu'exploiter une structure piano-convexe si finement agencée ne put s'inscrire dans l'urgence, ni dans l'adoption de moyens expédients. Ce projet qui dut être estival, exigea donc d'un artisan aux mains expertes, du temps et de l'espace, en plus de nombreuses connaissances mettant en œuvre une exploitation spécifique et organisée du derme ossifié, à des fins non ordinaires.

\section{Le plastron et la création de deux pendentifs (fig. 1 et 2)}

9 Le plastron (Roubet 1966, p. 235-237) est recouvert de six paires d'écussons épidermiques constituant l'écaille colorée en brun et or. L'os dermique ou derme ossifié sous-jacent se compose de 9 plaques osseuses, dont quatre paires de plaques articulées entre elles, et une plaque impaire centrale, interruptive de la suture médiane. Le plastron est relié au bouclier par deux paires d'hyoplastrons pourvues d'apophyses inguinales osseuses et se trouve soudé au bouclier par 5 paires de plaques marginales. Cinq étapes principales de préparation du plastron ont été reconnues, consistant à :

- dissocier le plastron du bouclier en découpant (sciage) les apophyses inguinales et en dessoudant les 10 plaques marginales;

- découper l'écaille du plastron dans le sens longitudinal et transversal à hauteur de l'hypoplastron (incision);

- dessouder les deux paires de plaques (xiphiplastron et hypoplastron) qui formeront chacune un pendentif, à droite et à gauche ;

- perforer le bord supérieur de chaque plaque supérieure (xiphiplastron) des deux pendentifs (perçoir et mêches) ;

- abraser les sutures longitudinales et transversales, aplanir la face interne des deux plaques (grés fin).

L'écaille si fragile recouvre parfaitement le plastron; les écussons sont imbriqués de manière à protéger et isoler les sutures sous-jacentes. Cette découpe initiale très délicate doit conserver l'adhérence de cette enveloppe cornée qui rehaussera le pendentif. L'écaille a certes une fonction esthétique, mais assure surtout la cohésion et le maintien des deux plaques. Lorsqu'elle vient à se décoller, on constate la dissociation des plaques et la perte de la plaque inférieure qui se détache de l'engrenure (retrouvée dans les gisements).

Deux pendeloques sont systématiquement élaborées à partir du même plastron constitué de deux paires postérieures de xiphiplastrons qui s'articulent aux hypoplastrons. L'artisan doit impérativement conserver deux plaques jointives pour chaque pendeloque. Seul le xiphiplastron reçoit une perforation pour la suspension.

De nombreuses contraintes techniques sont inhérentes à la taille de l'individu choisi : toutes les plaques étant d'assez petites dimensions, les dissocier, les découper, les perforer, exigent précision, sûreté du geste et finesse. D'autres obstacles se situent à l'engrenure qui peut s'ouvrir. Chaque zone de suture située à la périphérie d'une plaque (deux ou trois côtés), est fragile, dentée, symétrique, longitudinale et transversale, elle assure le raccord aux autres plaques du plastron; dessouder deux plaques reste donc un acte délicat à opérer sans endommager la partie symétrique. Le pendentif portant son écaille n'est achevé que lorsque toutes les sutures inutilisées ont été abrasées, 
émoussées pour former le contour lisse et sans aspérités, d'un bord arrondi et lorsque la face interne a été aplanie et que la périphérie de la perforation a été élimée (fig. 1 et 2). Parfois de l'ocre rouge imprègne encore le derme interne (Grotte Capéletti, troisième série d'occupation, Roubet 1979, p. 320).

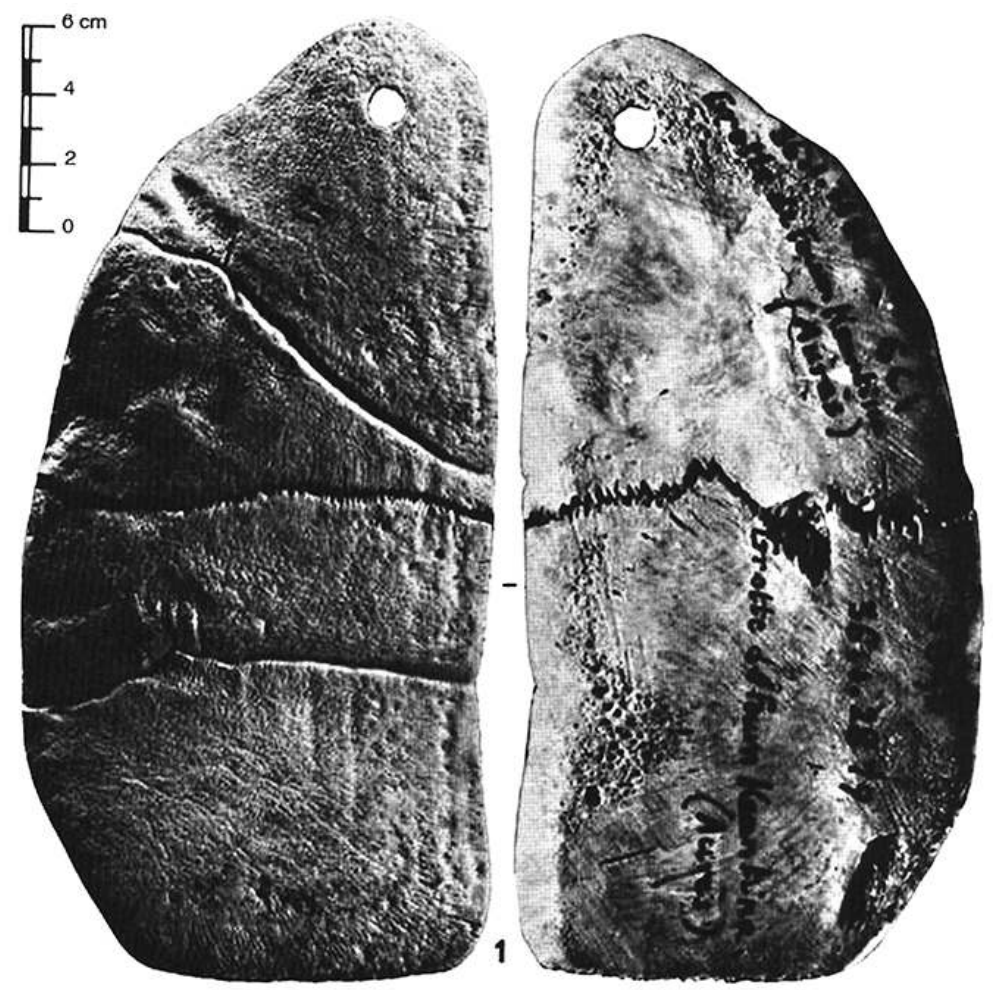

Fig. 1. PENDELOQUE déCOUVERTE DANS LA gROTTE CAPELETTI (AURÈS) IN ROUBET 1979, P. 127 ET 246. LA PARTIE SUPÉRIEURE PORTANT L'ORIFICE POUR UNE SUSPENSION EST UN XIPHIPLASTRON, ELLE A ÉTÉ RACCORDÉE À LA PARTIE INFÉRIEURE QUI EST UN HYPOPLASTRON. ON OBSERVERA LES MARQUES D'ABRASION SUR LA FACE INTERNE ET SUR LA PÉRIPHÉRIE (À DROITE), ALORS QUE SUR LA FACE EXTERNE LE DERME OSSIfIÉ, PRIVÉ DE SON ÉCAILLE (À gAUCHE) EST RESTÉ INTACT. CE DOCUMENT PROVIENT DES DÉPÔTS DE LA SECONDE SÉRIE D'OCCUPATION PASTORALE. LE RACCORD DES DEUX

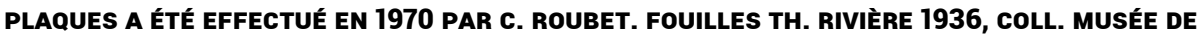
L'HOMME, PARIS.

Cl. J.-P. Kaufman (1970). 


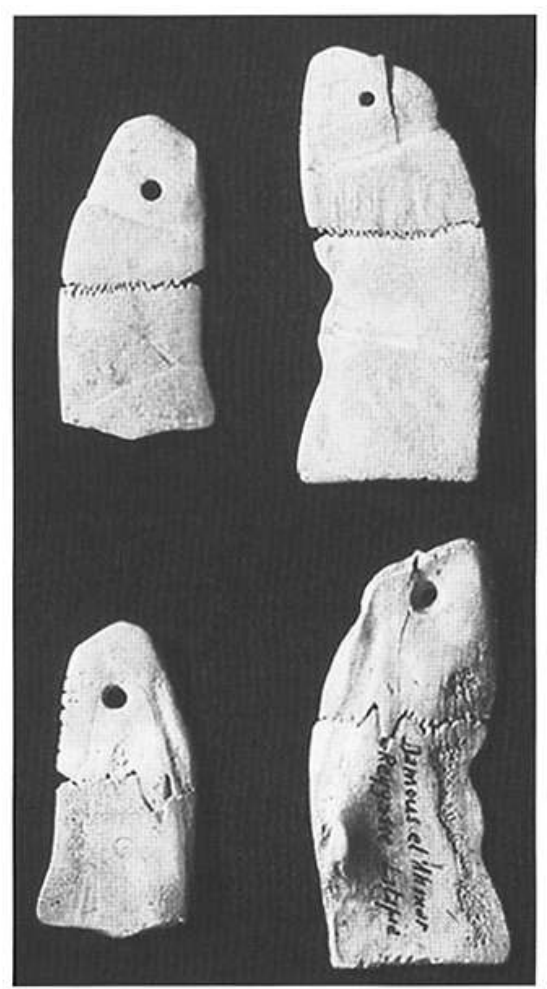

Fig. 2 : PENDELOQUES déCOUVERTES AU DAMOUS EL-AHMAR (NÉMENCHA) IN ROUBET 1966, P. 248-249. LE RACCORd DES PLAQUES DE CES DEUX PENDENTIFS A ÉTÉ RÉALISÉ PAR C. ROUBET EN 1964. FOUILLES LATAPIE-REYgASSE 1912, COLL. CNRPAH, ALgER).

Cl. F.-E. Roubet (1964).

\section{Le bouclier et la création d'une coupe (fig. 3 et 4)}

13 Le bouclier (Roubet 1966, p. 237-239), partie dorsale de la cuirasse osseuse, forme une solide enveloppe protectrice de 50 plaques (10 neurales; 8 paires costales; une couronne de 24 plaques marginales, dont 10 sont soudées au plastron par les paires d'apophyses inguinales). Plusieurs rangées d'écussons épidermiques recouvrent l'os dermique d'une écaille brillante et bicolore, symétriquement disposée par rapport à l'axe longitudinal médian. Conserver l'adhérence de cette écaille aux plaques sousjacentes assure le bon maintien des deux matériaux et contribue à entretenir une certaine imperméabilité externe à l'objet à façonner, qui est ici une coupe. Pour s'engager dans ce second projet une autre chaîne opératoire a été nécessaire, adaptée à la structure anatomique du bouclier et à la morphologie finale de la coupe. Cinq principales étapes de transformation du bouclier en coupe ont été reconnues consistant à :

- dessouder les 10 plaques marginales bordant la carapace ;

- araser les structures saillantes (reste d apophyse) de la face interne du bouclier : c'est-à-dire les apophyses épineuses de 8 vertèbres dorsales, ainsi que les côtes correspondantes (palet en grés fin) ;

- déchiqueter et gratter les tissus mous (tendons) adhérents au bouclier (silex). De multiples séries d'incisions transversales et obliques l'attestent; elles ont été produites par un outil lithique tranchant (fig. 4) ; 
- abraser les sutures vives apparues après le retrait des plaques marginales périphériques (plaques en grés fin);

- polir le nouveau rebord constitué par l'extrémité abrasée des plaques costales.

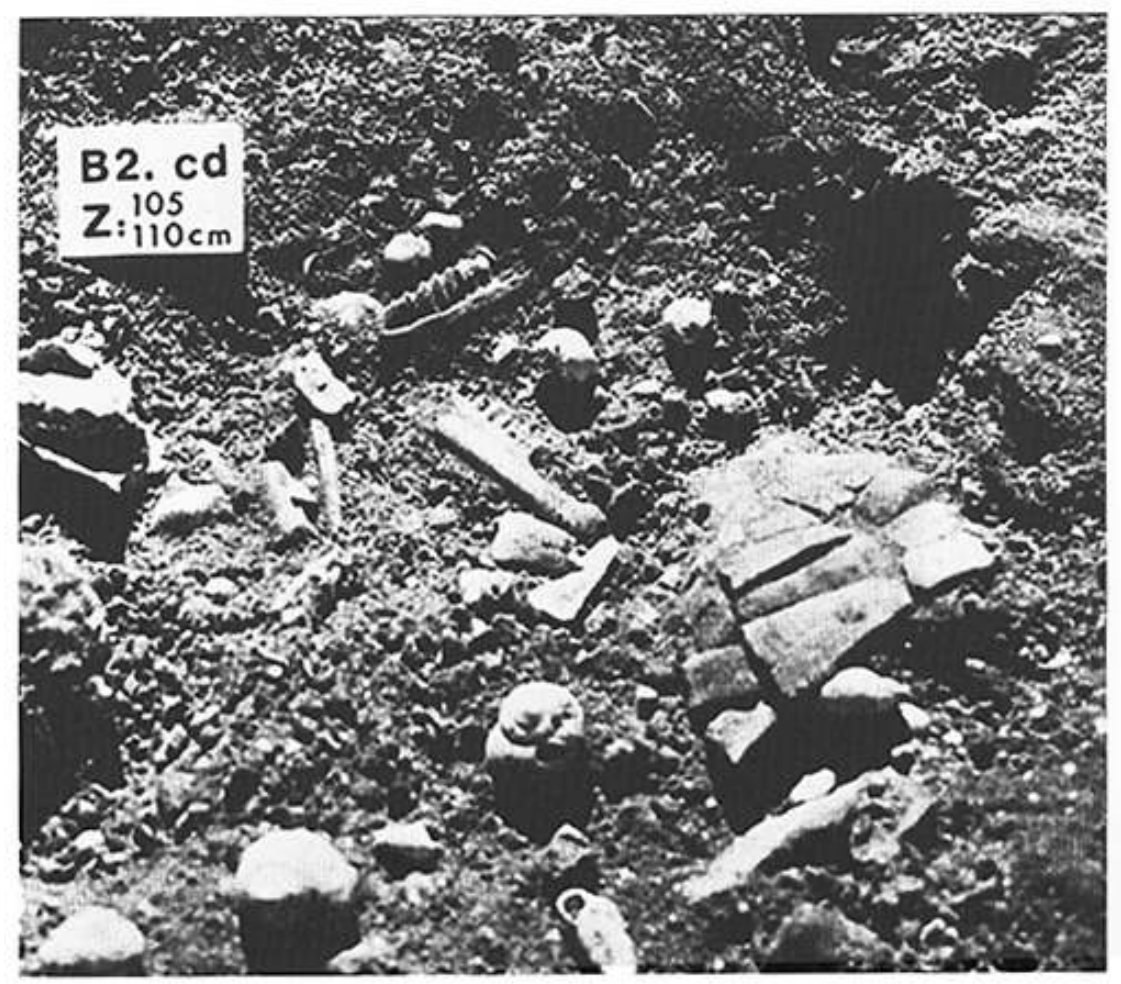

Fig. 3 : GROTTE CAPELETTI (AURÈS) : DÉCOUVERTE D'UNE COUPE. IN ROUBET 1979 P. 148 ET 314. FAÇONNÉE DANS UN BOUCLIER DE TORTUE TERRESTRE, CETTE COUPE PROVIENT DES DÉPÔTS IN SITU DE LA TROISIÈME SÉRIE D'OCCUPATION PASTORALE. LA COUPE EST RENVERSÉE ET ASSOCIÉE À DIVERS RESTES DE NOURRITURE.

Fouilles et cliché C. Roubet 1968 Coll CNRPAH, Alger.

Une coupe est achevée lorsque ne subsiste plus aucune aspérité périphérique et qu'un véritable bord arrondi et lisse en constitue le pourtour. La face interne, concave et sans aspérité, est alors celle d'une coupe. Parfois de l'ocre rouge imprègne encore le derme interne (Grotte Capéletti, troisième série d'occupation, Roubet, 1979, p. 315).

Pendentif-amulette et coupe portant encore de l'ocre ne participèrent pas aux tâches d'un quotidien. On peut leur reconnaître un rôle spécial, celui d'objets de prestige, voire d'éléments cérémoniels. 


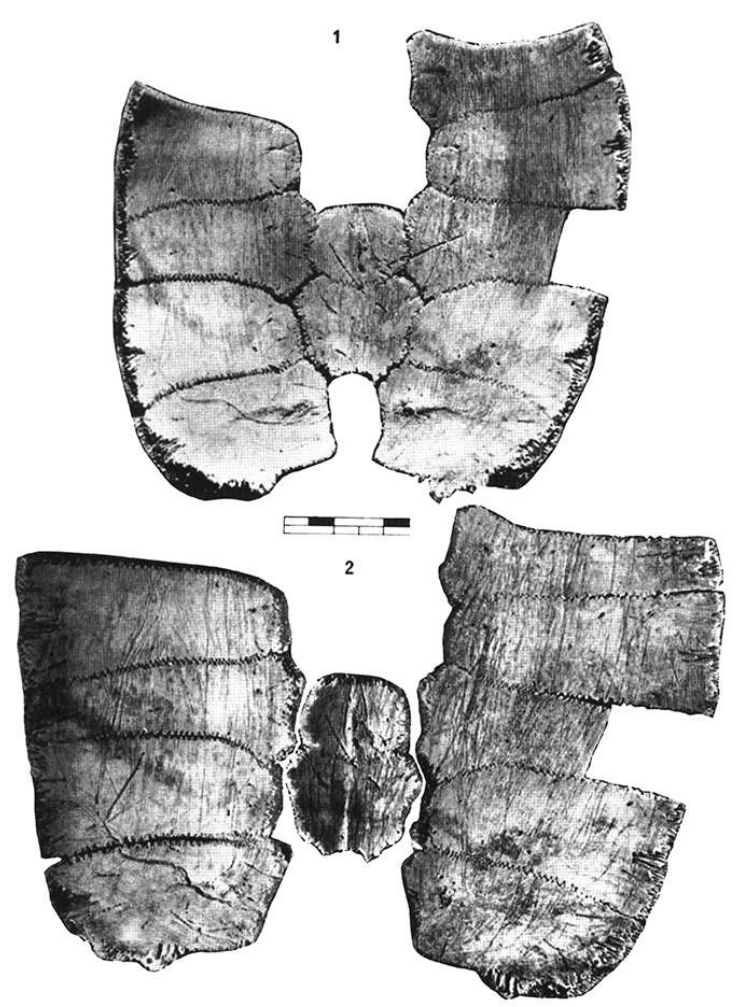

Fig. 4 : ReCONSTITUtion d'UNe COUPE DANS UN BOUCLIER DE TORTUE TERRESTRE. (ROUBET 1979), FOUILLES ET TRAVAUX C. ROUBET 1968, COLL. CNRPAH, ALgER.

\section{Objets de prestige ( ?)} terrestre, non transposable à d'autres matériaux, ne relève ni d'activités expédientes, quotidiennes ni de répertoires techniques usuels, ni d'une compétence générale acquise à partir du traitement de l'os. On a pu reconnaître l'existence d'une «industrie de l'os dermique » très élaborée et spécifique (Roubet 1979, p. 321) innovante, sans antécédents antérieurs. La finalité atteinte après tant de précautions prises, de gestes ajustés, de patience entretenue dans l'isolement exigé, se réduisit-elle à n'obtenir que deux pendeloques recouvertes d'écaille et une coupe au bord régulier? Conclusion objective, triviale. Qui furent donc ces experts aux mains d'artiste, présents dans tant d'autres régions montagneuses, de la Kroumirie* à la Dorsale tunisienne, du Constantinois, au Babors, à l'Oranie littorale, des Némencha aux Monts des Ksour, puis au Maroc atlasique? Pourquoi se sont-ils contraints à façonner avec tant de soin ces créationslà ? Seuls de sages spécialistes installés à l'écart d'un quotidien ont pu entreprendre et réaliser de telles créations selon des codes anatomiques et techniques, maîtrisés ; on ne saurait donc rapprocher ces pendentifs-amulettes, parfois ocrés, d'autres parures habituelles (en pierre), pas plus qu'on ne saurait considérer la coupe, contenant parfois un mélange d'ocre, comme un ustensile usuel (en céramique). Les multiples connaissances acquises, écologiques, éthologiques et techniques inciteraient à installer l'usage de ces deux objets dans un cadre cérémoniel, selon un rituel resté insaisissable. Leur rareté, leur dispersion, semblent indiquer une diffusion accompagnant le pastoralisme montagnard (Roubet 2001a, 2010a). 


\section{BIBLIOGRAPHIE}

CAMPS-FABRER H., 1960 - « Parure des temps préhistoriques en Afrique du Nord », Libyca, VII, p. 11-218.

CAMPS-FABRER H., 1966 - Matière et art mobilier dans la Préhistoire nord-africaine et saharienne, Paris, AMG (Mémoire du CRAPE, Alger : V), 574 p.

ROUBET C., 1966 - «L Les pendeloques en plaques dermiques de tortue dans le Néolithique de l'Afrique du Nord », Libyca, XIV, p. 223-259.

ROUBET C., 1968 - Le gisement du Damous el Ahmar et sa place dans le Néolithique de tradition capsienne, Paris, AMG (Travaux du CRAPE, Alger), 144 p.

ROUBET C., 1979 - Economie pastorale préagricole en Algérie orientale : le Néolithique de tradition capsienne. Exemple L'Aurès, Paris, CNRS (Etudes d'Antiquités Africaines), 595 p.

ROUBET C., 2001a - « Le Néolithique de Tradition Capsienne sensu strict », Encyclopedia of Prehistory. Volume 1: Africa, 10 entrées, in "Outlines of Archaeological Traditions. The Human Relations Area Files”, Ed. by P.-N. Peregrine and M. Ember, Kluwer Academic, Plenum Publishers, p. 197-219.

ROUBET C., 2003 - « "Statut de berger" des communautés atlasiques néolithisées du Maghreb oriental », L'Anthropologie, t. 107, p. 393-442.

ROUBET C., 2006 - « Pastoralisme et ruralité néolithiques dans l'Aurès, avec la grotte Capéletti, entre $7500 \mathrm{cal} \mathrm{BP}$ et $5400 \mathrm{cal} \mathrm{BP} \mathrm{»,} \mathrm{Aouras,} \mathrm{3,} \mathrm{p.} \mathrm{19-35.}$

ROUBET C., 2010 - « Statut social des pasteurs néolithiques des Némencha : Objets de prestige », Aouras, 6, p. 39-55. 
INDEX

Mots-clés : Aurès, Capsien, Néolithique, Néolithisation, Parure, Sahara, Zoologie 